\title{
Verbraucherpreise im Zeichen multipler Sonderfaktoren
}

Die Teuerung auf der Konsumentenstufe hat sich zum Jahresauftakt merklich beschleunigt. Legte der Verbraucherpreisindex (VPI) im Dezember 2020 im Vormonatsvergleich nur um 0,2\% zu, wurde im Januar 2021 mit 1,3\% der stärkste Anstieg seit fast 30 Jahren verzeichnet. Noch ausgeprägter zeigt sich der Teuerungsschub im Harmonisierten Verbraucherpreisindex (HVPI), der im Januar um $1,7 \%$ zulegte. ${ }^{1}$ Auch im Vorjahresvergleich zeigt sich zum Jahresauftakt eine höhere Inflationsdynamik: Waren die Konsumentenpreise ab Mitte 2020 rückläufig, zogen sie im Januar und Februar im Zwölfmonatsvergleich in beiden Messkonzepten mit steigender Tendenz um etwa $1 \%$ an. Auch wenn dies erst der Auftakt zu im weiteren Jahresverlauf steigenden Inflationsraten sein dürfte, so ist darin noch nicht der Eintritt in ein neues Inflationsregime zu sehen.

Der Auftrieb der Verbraucherpreise speist sich im Verlauf von 2021 aus einem ganzen Bündel von Sonderfaktoren. Der Preisschub zum Jahresauftakt spiegelt im Wesentlichen die Folgen gesetzgeberischen Handelns wider. So erhöht die Rückkehr zu den vormaligen Mehrwertsteuersätzen (mit Ausnahme des bis Ende 2022 weiterhin ermäßigten Satzes auf Speisen in der Gastronomie) für sich genommen die Verbraucherpreise um 1,2\%. Hierbei ist eine Überwälzung von zwei Dritteln unterstellt (Ademmer et al., 2020, 11). Der im zurückliegenden Halbjahr wirksame inflationsdämpfende Effekt entfällt somit in den ersten sechs Monaten des laufenden Jahres. Ab der Jahresmitte 2021 macht sich die Normalisierung der Mehrwertsteuer-

(c) Der/die Autor:in(nen) 2021. Open Access: Dieser Artikel wird unter der Creative Commons Namensnennung 4.0 International Lizenz veröffentlicht (creativecommons.org/licenses/by/4.0/deed.de).

Open Access wird durch die ZBW - Leibniz-Informationszentrum Wirtschaft gefördert.

1 Während dem als Laspeyres-Index konstruierten VPI unverändert der Warenkorb des Jahres 2015 zugrunde liegt, spiegelt sich in dem nach Paaasche konzipierten HVPI auch die Umstellung des Warenkorbs auf das Jahr 2021 wider. Da es im Zuge der Pandemie zu teils erheblichen Umschichtungen in den Ausgabeanteilen einzelner Gütergruppen kam, erklärt dieser Kompositionseffekt einen Großteil der Diskrepanz in der Inflationsmessung zwischen VPI und HVPI. Insbesondere ergibt sich ein Sondereffekt aufgrund des ausgeprägten Saisonmusters der Komponente Pauschalreisen und der Verkettung des HVPI, durch die (imputierte) Preisanstiege im Jahresverlauf bis Dezember mit dem alten, höheren Gewicht, der Rückgang von Dezember auf Januar aber nur mit dem neuen, niedrigeren Gewicht eingerechnet werden. Dieser Sondereffekt ist nur vorübergehender Natur und wird sich im späteren Verlauf umkehren (Deutsche Bundesbank, 2021). sätze abermals in der monatlichen Inflationsrate bemerkbar, weil in den Vorjahresvergleich dann aktuelle Preise (mit normalen Mehrwertsteuersätzen) auf Vorjahrespreise (mit reduzierter Mehrwertsteuer) eingehen. Bezogen auf das Gesamtjahr erhöht der Mehrwertsteuereffekt die Inflationsrate um 0,6 Prozentpunkte. Zusätzlich traten zu Jahresbeginn höhere $\mathrm{CO}_{2}$-Steuern in Kraft, die für sich genommen das Preisniveau im laufenden Jahr um 0,4\% anheben.

Der massive Einbruch der wirtschaftlichen Aktivität hat in den ersten Monaten des vergangenen Jahres zu einem Preissturz bei Energierohstoffen geführt. Der entsprechende HWWI-Rohstoffpreisindex hat sich von Dezember 2019 bis April 2020 mehr als halbiert und lag am Jahresende immer noch $23 \%$ unter dem Vorkrisenniveau. Mit der mittlerweile fortgeschrittenen Erholung der Energiepreise kommt es auch von dieser Seite das ganze Jahr über zu erheblichen Basiseffekten, die die monatlichen Inflationsraten entsprechend höher ausfallen lassen. Für sich genommen dürften $40 \%$ höhere Energierohstoffpreise die Inflationsrate schätzungsweise um 0,4 Prozentpunkte anheben.

Wenig hilfreich für die Analyse der Preisdynamik sind in 2020 und 2021 die Schätzungen zur gesamtwirtschaftlichen Produktionslücke. So ist der Phillips-KurvenZusammenhang seit geraumer Zeit ohnehin nur noch schwach ausgeprägt (Deutsche Bundesbank, 2016), und die Natur der Corona-Krise lässt in dieser Hinsicht sogar noch schwächere Effekte erwarten. Denn - insbesondere mit Blick auf die konsumnahen Wirtschaftsbereiche - ist der Einbruch der Wirtschaftsleistung nicht durch eine allgemeine Nachfrageschwäche gekennzeichnet, sondern dadurch, dass ökonomische Aktivität aus Infektionsschutzgründen unterblieb. Diese lässt sich von den Unternehmen nicht durch Preisnachlässe anregen. Dies gilt insbesondere dort, wo Geschäftsmodelle durch Lockdown-Anordnungen unterbunden wurden. Zudem schmälert dies insgesamt die Präzision der Preismessung, da die statischen Ämter bei ausbleibenden Transaktionen auf Zuschätzungen angewiesen sind (Statistisches Bundesamt, 2021a).

Die größte Unbekannte für die weitere Entwicklung der Verbraucherpreise liegt in der zusätzlichen Ersparnis, die die privaten Haushalte während der Pandemie bilden. 
Abbildung 1

\section{Sparverhalten der privaten Haushalte}

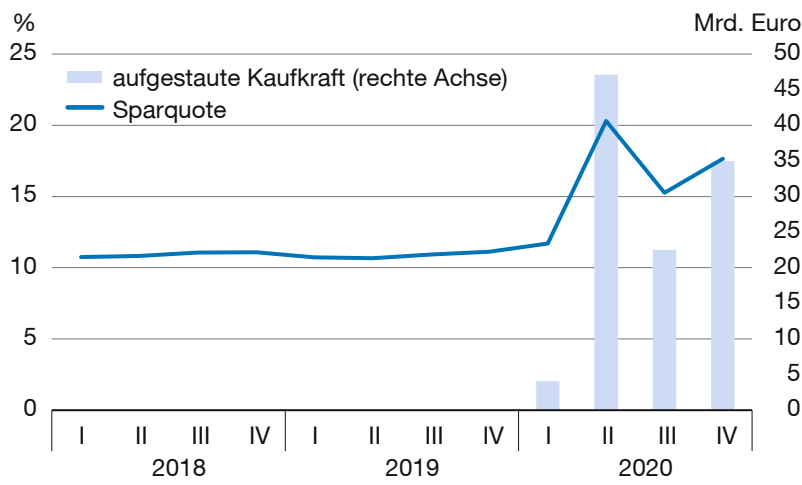

Anmerkungen: Quartalsdaten, saisonbereinigt. Sparquote: Sparen der privaten Haushalte und Organisationen ohne Erwerbszweck in \%; aufgestaute Kaufkraft: Differenz der Ersparnis gegenüber hypothetischer Sparquote von $10,9 \%$ in Mrd. Euro.

Quelle: Statistisches Bundesamt (2021b); Berechnungen des IfW Kiel.

Darin kommt zum Ausdruck, dass die Konsumenten ihre gewohnten Ausgabemuster (z. B. für Gaststättenbesuche oder Urlaubsreisen) aufgrund von behördlichen oder privaten Infektionsschutzmaßnahmen nicht wahrnehmen können und ihre Einkommen kurzfristig auch nicht in andere Verwendungsarten umschichten. Insgesamt ist so allein im vergangenen Jahr Kaufkraft von fast 110 Mrd. Euro aufgestaut worden (vgl. Abbildung 1). Im laufenden Jahr könnten weitere $85 \mathrm{Mrd}$. Euro hinzukommen (Ademmer et al., 2020, 8 f.). Zusammengenommen entspricht dies gut $10 \%$ der gesamten privaten Konsumausgaben eines Jahres. Hierzu trägt maßgeblich bei, dass staatliche Transfers die privaten verfügbaren Einkommen weitgehend von den Folgen des Produktionseinbruchs abschirmen. So ging die Wirtschaftsleistung 2020 (gemessen am nominalen Bruttoinlandsprodukt) um 3,4\% zurück, während die verfügbaren Einkommen der privaten Haushalte um 0,7\% leicht zunahmen. Die Masseneinkommen (im Wesentlichen Nettolöhne und monetäre Sozialleistungen) nahmen sogar mit 2,6\% merklich zu. Die nominalen privaten Konsumausgaben brachen demgegenüber um $5,4 \%$ ein.

Sobald die bislang aus Infektionsschutzgründen gesperrten Konsumzweige wieder offen stehen, dürfte die Nachfrage nicht nur rasch wieder auf das Vorkrisenniveau zurückkehren, sondern es in den während der Pandemie entbehrten Bereichen auch zu Nachholeffekten kommen, die tendenziell preistreibend wirken. Unklar ist, in welchem Umfang die privaten Haushalte dabei auf die aufgestaute Kaufkraft zurückgreifen werden. Für die Preiseffekte spielt auch eine Rolle, wie viele Unternehmen bis zum Wegfall der Corona-bedingten Einschränkungen aufgeben werden bzw. wie schnell neue Unternehmen an
Abbildung 2

Verbraucherpreisinflation

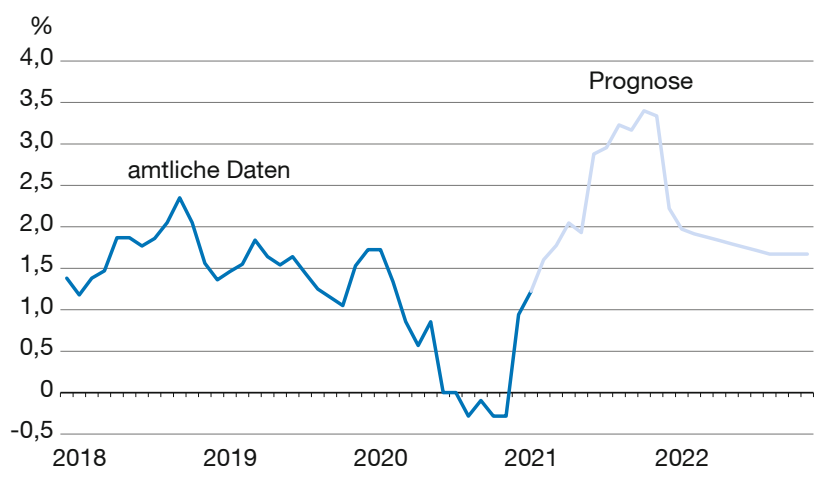

Anmerkungen: Monatsdaten (letzter Ex-post-Wert: Februar 2021). Veränderung des Verbraucherpreisindex (VPI) gegenüber dem Vorjahr in \%.

Quelle: Statistisches Bundesamt (2020); Prognose des IfW Kiel.

ihre Stelle treten. Für 2021 und 2022 stellen Nachholeffekte der Konsumenten daher ein erhebliches Aufwärtsrisiko für die Inflation dar.

All diesen Faktoren ist gemein, dass sie die Inflationsrate nur vorrübergehend steigen lassen. Für 2021 rechnen wir derzeit mit einer Verbraucherpreisinflation von 2,4\%; in der unterjährigen Spitze dürften die Vorjahresraten sogar die 3\%-Marke reißen (vgl. Abbildung 2). Spätestens im Verlauf des kommenden Jahres bilden sich die Raten wieder zurück - 2022 wird die Inflation wohl wieder weniger als $2 \%$ betragen. Als Vorbote zu im Trend deutlich höheren Inflationsraten als vor der Corona-Krise kann der Preisschub im laufenden Jahr daher nicht gelten.

Salomon Fiedler, Stefan Kooths stefan.kooths@ifw-kiel.de

\section{Literatur}

Ademmer, M., J. Boysen-Hogrefe, S. Fiedler, D. Groll, N. Jannsen, S. Kooths und S. Meuchelböck (2020), Deutsche Wirtschaft im Winter 2020: Zweite Corona-Welle unterbricht Erholung, Kieler Konjunkturberichte, 74 .

Deutsche Bundesbank (2016), Die Phillips-Kurve als Instrument der Preisanalyse und Inflationsprognose in Deutschland, Monatsbericht, April, 31-45.

Deutsche Bundesbank (2021), Monatsbericht, Februar, 64-67.

Statistisches Bundesamt (2020), Fachserie 18, Reihe 1.3.

Statistisches Bundesamt (2021a), Zu den Auswirkungen der CoronaKrise auf die Preiserhebung für den Verbraucherpreisindex/Harmonisierten Verbraucherpreisindex, https://www.destatis.de/DE/Themen/ Wirtschaft/Preise/Verbraucherpreisindex/Methoden/Downloads/ corona-vpi-hvpi.pdf;jsessionid=E2CA457955E04AE1F4C7F86221DF 14B5.internet721?__blob=publicationFile (8. März 2021).

Statistisches Bundesamt (2021b), Fachserie 17, Reihe 7. 